\title{
Milk and Milk Products - Good or Bad
}

\author{
Anil Kumar \\ School of Biotechnology, Devi Ahilya University, Khandwa Road, Indore-452001, India \\ Email: ak_sbt@yahoo.com
}

\begin{abstract}
Since long time, it has been considered that milk and milk products are healthy foods for human beings. On the other hand, some milk products have been reported to exhibit harmful effects. There are reports that milk and other dairy products when consumed by human beings especially in higher amount increase the risk of many chronic diseases like obesity, type 2 diabetes, cardiovascular diseases, osteoporosis and even cancers of various types. A large number of cohort studies on large and variable population of humans have been carried out for different diseases and data are analyzed using multivariable statistical models. High fat contents containing milk products, presence of carcinogenic pesticides and/or growth factors like IGF-1 may promote cancer cell growth. However, no conclusive evidence is there for harmful effect of milk and its products. In spite of no conclusive evidence for harmful effects, nowadays, many people especially in developed countries prefer plant derived drinks isolated from rice, soybean, almonds etc in place of milk and its products.
\end{abstract}

Keywords: Milk, milk (Dairy) products, bone integrity, pesticides, breast cancer, fracture, cardiovascular diseases, anthropometric variables, adiposity, lung cancer, odd carbon fatty acids, diabetes

\section{Introduction}

Nowadays, there is much debate on the health effects of dairy products in the common public especially in developed countries and more and more people are preferring plant derived alternate products/ drinks isolated from rice, soybean, almonds, oats etc. Many scientists are engaged in researches related to dairy products consumption and possible risk of diseases like diabetes, obesity, cardiovascular diseases, osteoporosis, cancer, and all-cause mortality (Thorning et al, 2016). There are number of reviews and studies supporting positive health effects of dairy products. Nutritionists suggested lesser risk of obesity during childhood upon intake of milk and its products. Studies also showed a positive correlation in adults between intake of milk and its products and improvement of body composition. These products also help in carrying weight loss during low energy uptake. Studies are also in favor of positive correlation between milk and other dairy products intake and reduced risk of diabetes and cardiovascular disease especially stroke. After reviewing the studies, Thorning et al (2016) concluded that milk and other dairy products intake are inversely related with colorectal cancer, bladder cancer, gastric cancer, ovarian cancer and lung cancer and evidences for prostate cancer are inconsistent. They also indicated that milk and other dairy products are not responsible for all-cause mortality. Cow milk and plant based drinks do not seem to be equivalent. More researches must be carried out for checking equivalence of plant based drinks with dairy products.

\section{Advice to Women and Doctors about Milk}

Horwath et al (1995) conducted a study on elderly women aged more than 70 years old as well as on younger women students of New Zealand who were low calcium consumers. They found that elder women were mostly of the view that higher milk or other dairy products consumption would have either no effect on their health or negative effects on certain diseases like diabetes, high blood pressure, high cholesterol and heart diseases. However, younger women students were aware of potential health benefits if they intake higher milk quantity compared to other milk products. Contrarily, some younger women were afraid of bad impact on higher consumption of milk since they believed that they will gain more body weight by consuming milk. It was also found that nearly 13 to $18 \%$ women were having 
advice from the doctors not to consume milk or milk products for different reasons. It was also found that in both age groups, many women were not liking milk consumption in addition to health reasons. After reviewing all the facts, they concluded that there is need of counselling to both women as well as doctors for telling beneficial effects of milk consumption. There is need of convincing the youngsters that for keeping weight control, there is no need of excluding milk and milk products from the daily routine diet. There is need of awareness programs to convince about the beneficial aspects of low fat milk and milk products especially for the bones health.

\section{Milk / Milk Products and Cancer}

In spite of much research being carried out in the field of cancer biology, cancer is still considered to be a deadly disease. As per report of World Health Organization (WHO), there were nearly 9.6 million deaths globally due to various types of cancers and it is considered to be the second leading cause of deaths globally. Out of these, more death cases are reported from low and medium income group countries. The reported risk factors for cancer are genetic inheritance, diet, smoking, physically inactiveness, environmental pollution, radiations etc. On an average, 90 to $95 \%$ cancers are developed due to various environmental factors or improper diet (Anand et al, 2008). As per estimate, nearly 35\% cases of cancer are due to improper diet intake (Baena et al, 2014).

When we talk about milk and milk products in the diet, these are considered to be beneficial components of the diet since long time. It is considered that dairy products are much important for good healthy life. However, there are certain reports where harmful effects of dairy products have been reported. There are reports that harmful reproductive and cancer causing hormones are present in milk and its products (Qin et al, 2004; Maruyama et al, 2010). Renehan et al (2004) reported that regular consumption of milk and its products may cause cancer due to increase in insulin like growth factor levels. Jeyaraman et al (2019) overviewed the reviews and case studies published between 1991 and 2017 on the aspect of cancer risk due to milk and milk products and performed systematic statistical analyses. The analyses indicated that hormone dependent cancers viz. prostate cancer, ovarian cancer, breast cancer and endometrial cancer may be developed upon consumption of milk and milk products. Contradictory reports are present where it has been shown that dairy products viz. milk, skim milk, yogurt, cheese, butter or ice cream consumption does not cause any significant or decreased risk of prostate cancer. However, on the other hand, reports of increased risk of prostate cancer with more consumption of milk and milk products viz. milk, low fat milk, cheese or dairy calcium are there (Jeyaraman et al, 2019; Huncharek et al, 2008; Qin et al, 2007; Epstein et al, 2011). Similarly, there are reports on increased risk of ovarian cancer upon higher uptake of whole milk or lactose (Larsson et al, 2006; Genkinger et al, 2006; Liu et al, 2015). Filomeno et al (2015) reported positive correlation between consumption of all dairy products and endometrial cancer. Boyd et al (1996) indicated increased risk of breast cancer upon higher intake of milk or cheese in the diet by women.

\section{Milk, Calcium and Bone Integrity}

It is recommended in many nutrition policies that daily consumption of 800 to $1500 \mathrm{mg}$ of calcium helps in prevention of osteoporosis and calcium is mostly taken in the form of milk and other dairy products. Lanou et al (2005) reviewed the research papers to correlate the effect of dairy products and total calcium intake on bone integrity in children and young adults, and whether dairy products are better for bone integrity than other calcium enriched foods or supplements. They analyzed a total 58 studies taken from the literature. Out of these 58 studies, eleven studies did not control for weight, puberty status and exercise and therefore were removed from the analysis. Ten studies were randomized having controlled trials of supplemental calcium and in these out of 10 studies, nine indicated modest positive benefits on bone integrity in children as well as young adults. In nine studies, smaller health benefits were found correlated with dairy calcium intake. In three studies, vitamin D was also present with calcium since they used fortified milk with vitamin D. Ultimately, they concluded that only little evidences are there which correlated higher dairy products intake with bone mineralization/ integrity in children as well as young adults. 


\section{$5 \quad$ High Fat Milk, Pesticides, Breast and Prostate Cancers}

Moorman and Terry (2004) gave different reasons for increased risk of breast cancer after consumption of dairy products namely presence of high total fat contents, saturated fat, presence of the pesticides which may be carcinogenic, presence of growth factors like IGF-1 which promotes cancer cell growth. On the other hand, it has been said that some constituents of dairy products viz. calcium, vitamin D, rumenic acid, butyric acid, branched chain fatty acids and whey protein protect against breast cancer (Moorman and Terry, 2004; Parodi, 2005).

It has been speculated that intake of high fat diet including high fat milk and other dairy products increases the probability of development of some cancers like breast, colon and prostate cancers. However, after carrying much analyses, Food and Agricultural Organization of United Nations (FAO) and World Health Organization (WHO) concluded that no probable or convincing evidences are there to prove significant effects of total fat present in diet on cancer. FAO and WHO also found no significant evidence to prove positive relationship between consumption of saturated fatty acids and cancer. However, on the other hand, on the basis of available data, FAO and WHO found a positive correlation between total dietary fat and obesity and overweight. According to World Cancer Research Fund (WCRF) and American Institute for Cancer Research (AICR), there is increased risk of cancers of rectum, colon, oesophagus, endometrium, pancreas, kidney and breast in persons with obesity, and/ or overweight. There is no direct evidence indicating that milk or milk products cause cancer. In fact, it has been shown that some constituents in milk viz. calcium, vitamin D, sphingolipids, proteins, butyric acid act as protective agents against cancers (Weaver et al, 2013).

\section{Milk, Milk Products and Risk of Fracture/Mortality}

On the basis of cohort studies, Michaelsson et al (2014) investigated correlation between consumption of milk and risk of mortality and fractures in women and men. They selected a group of 61,433 women aged between 39 to 74 years at baseline 1987 to 90, and another group of 45,339 men aged between 45 to 79 years at baseline 1997. They used multivariable survival models to determine the association between milk consumption and time to mortality or fracture. The results indicated that during a mean follow up of 20.1 years, there was death of 15541 women. However, 17252 women had a fracture and out of these, 4259 got hip fracture. The results with men indicated that with a mean follow up of 11.2 years, there was death of 10112 men and in addition 5066 men got fracture and out of these, 1166 got hip fracture. In women drinking three or more glasses of milk daily compared to women drinking less than one glass of milk, adjusted mortality hazard ratio was 1.93. For each glass of milk, adjusted mortality hazard ratio was 1.15 in women and 1.03 in men. They concluded that higher milk intake was associated with higher mortality in one cohort of women and in another cohort of men, and with higher fracture incidence in women.

\section{Milk / Milk Products and Cardiovascular Diseases}

Guo et al (2017) determined the risk of cardiovascular diseases and all-cause mortality on consumption of milk and other dairy products consumption by conducting a dose-response meta-analysis. They took the research articles published up to September, 2016 by searching various databases viz. PubMed, Embase, Scopus. They conducted random-effect meta-analyses with summarized dose-response data for high as well as low fat dairy products, milk, fermented dairy products, cheese, yogurt etc. They investigated non-linear associations using the spine models and heterogeneity by sub-group analyses. They found no association for high as well as low fat dairy products and milk with the health outcomes of mortality, coronary heart disease or cardiovascular disease. On the other hand, marginally inverse associations were found between total fermented dairy products like sour milk products, cheese or yogurt with mortality and cardiovascular disease risk. However, all these marginally inverse associations of totally fermented dairy products and cheese were weakened in sensitivity analyses after removing one large Swedish study. 


\section{Milk / Dairy Products and Anthropometric Variables/ Adiposity}

The current concept with respect to positive correlation for consumption of milk and other dairy products and weight increase, overweight, obesity etc is mainly based on inconclusive and contradictory results. Therefore, Schwingshackl et al (2016) studied the correlation between consumption of milk and milk products and anthropometric variables/ adiposity in adult people by using meta-analysis of cohort studies. For that, they searched all the research papers published up to May, 2016 by using databases viz. PubMed, Embase, Web of Sciences, Google scholar. They collected all the research papers published in any language or on any calendar date. They included all the cohort studies which reported about the change in body weight, waist circumference, overweight, obesity by intake of dairy products viz. milk, yogurt, cheese, butter. They calculated the pooled effects using a random effects model and also carried the sensitivity analysis using a fixed effect model. They performed the analyses separately for betacoefficients of changes in body weight and/ or waist circumference per serving of dairy product, for differences in weight increase/increase in waist circumference when comparing extreme categories of dairy consumption, and for odds ratios in regard to weight gain, obesity, or abdominal obesity. They found inverse association between changes in body weight for each serving's increase of yogurt, however, each serving's increase of cheese showed positive association. They also found that highest dairy intake category was associated with a reduced risk of abdominal obesity and risk of overweight compared to the lowest intake category. They concluded that further research is required since overall interpretation of the results is limited by heterogeneous risk estimates.

\section{Milk, Dairy Products and Lung Cancer}

Yang et al (2016) analyzed the data of the research articles published up to April 01, 2015 by using the databases viz. PubMed and Web of Sciences. They showed that after pooling the results of individual studies, the relative risks of lung cancer for the highest versus lowest intake was 1.05 and 1.08 for total dairy products and milk, respectively. They found the negative results on the consumption of cheese, yogurt and low fat milk. They found the relative risks for total and dietary calcium intakes as 0.99 and 0.85, respectively. They concluded that intake of dairy products or calcium was not statistically associated with the risk of lung cancer. They suggested further research to be carried out in order to find correlation between dietary components and lung cancer.

\section{Milk, Dairy Products, Odd Carbon Fatty Acids and Diabetes}

Imamura et al (2018) analyzed the prospective associations of circulating or adipose tissue odd carbon fatty acids (C15 and C17) and trans-palmitoleic acid with type 2 diabetes using these fatty acids as biomarkers of dairy fat. They carried harmonised individual level analysis for the prospective associations using sixteen prospective cohorts from twelve different countries including seven from the USA, seven from Europe, one each from Taiwan and Australia. A total 63682 persons of wide difference in age were evaluated. The study specific results were pooled using inverse- variance-weighted metaanalysis. The study showed higher levels of odd carbon fatty acids (C15 and C17) and trans-palmitoleic acid were associated with lower incidence of type 2 diabetes. The positive point in taking these fatty acids as biomarkers was that these do not differentiate between different dairy products like cheese, milk, yogurt etc. Their study indicated that higher levels of these biomarkers were associated with a lower risk of type 2 diabetes.

\section{Milk and Coronary Heart Diseases}

Professor Maciej Banach in his presentation at the annual Congress of European Society of Cardiology on August 28, 2018 held at Munich, Germany mentioned that it has been speculated since long time that consumption of dairy products especially having high saturated fat contents increases the risk of death especially from cerebrovascular disease, coronary heart disease and cancer. However, results on the studies are not consistent except of milk which has been found to increase the risk of coronary heart 
disease. He suggested that guidelines to limit consumption of dairy products, especially cheese and yogurt must be relaxed and simultaneously consumption of low fat or fat free milk must be recommended (https://www.escardio.org/The-ESC/Press-Office/Press-releases/Current-advice-to-limitdairy-intake-should-be-reconsidered).

\section{Prospective Urban Rural Epidemiology (Pure) Study}

Dehghan et al (2018) carried the Prospective Urban Rural Epidemiology (PURE) study for determining the association of dairy intake with cardiovascular disease and mortality in 21 countries from five different continents by analyzing a cohort study. They took milk and other dairy products consumption data divided into low fat and whole fat dairy products of 136384 persons aged between 35 to 70 years from 21 different countries. They analyzed for the composite of mortality or deaths from cardiovascular causes, non-fatal myocardial infarction, stroke or heart failure. For hazard ratios, multivariable Cox frailty models with random intercepts was used. The results indicated that dairy products consumption was associated with lower risk of mortality and major cardiovascular disease events.

\section{Milk, Dairy Products and Hypertension}

Pouliot (https://www.dairynutrition.ca/scientific-evidence/experts-summaries/the-benefits-of-milkproducts-on-blood-pressure-a-role-for-bioactive-peptides) explained that hypertension mainly increases the chances of cardiovascular diseases and disability and predicted it to be the main factor for deaths due to cardiovascular diseases and disability globally by 2020. He also speculated that alone in Canada, more than five million people are suffering from hypertension (high blood pressure) and situation may be epidemic since probability is that $90 \%$ people in Canada will develop hypertension if they do not change to healthy lifestyle. He recommended consumption of diet enriched in fruits, vegetables, low fat milk products (DASH diet) which can control hypertension. Milk protein casein has specific bioactive peptides which have angiotensin-1-converting enzyme inhibitory effect, an important aspect for controlling blood pressure. The peptides, isoleucine-proline-proline and valine-proline-proline are reported to exhibit antihypertensive activity.

\section{Conclusion}

There are scare evidences that upon consumption of high fat milk and milk products, person may suffer by many diseases like cardiovascular disease, coronary heart disease, type 2 diabetes, cancer, osteoporosis etc. On the other hand, there are bioactive peptides present in milk protein which are helpful in controlling hypertension. People must prefer to take low fat milk and its products. More researches are required to reach on a firm conclusion.

Acknowledgements. Author acknowledges the facilities of the Department of Biotechnology, Ministry of Science and Technology, Government of India, New Delhi (DBT) present in the Department and used in this study which have been provided under the Distributed Bioinformatics Sub Centre and M.Sc. Biotechnology program.

\section{References}

1. Anand, P., Kunnumakkara, A. B. and Kunnumakara, A. B. et al. (2008) Cancer is a preventable disease that requires major lifestyle changes. Pharm Res 25, 2097-2116.

2. Baena- Ruiz, R. and Salinas- Hernández, P. (2014) Diet and cancer: risk factors and epidemiological evidence. Maturitas 77, 202-208.

3. Boyd, N. F., Martin, L. J. and Noffel M. et al. (1993) A meta-analysis of studies of dietary fat and breast cancer risk. Br J Cancer 68, 627-636. 
4. Dehghan, M., Mente, A., Rangarajan, S., Sheridan, P., Mohan, V., Iqbal, R. et al (2018) Association of dairy intake with cardiovascular disease and mortality in 21 countries from five continents (PURE): a prospective cohort study. Lancet 392, 2288-2297.

5. Epstein, M. M. and Smith-Warner, S. A. (2011) Risk of prostate cancer with intake of dietary and total calcium: A pooled analysis of 14 prospective cohort studies. Cancer Prevention Research Conference: AACR International Conference on Frontiers in Cancer Prevention Research 4.

6. Filomeno, M., Bosetti, C. and Bidoli, E. et al. (2015) Mediterranean diet and risk of endometrial cancer: a pooled analysis of three Italian case-control studies. Br J Cancer 112, 1816-1821.

7. Genkinger, J. M., Hunter, D. J. and Spiegelman, D. et al. (2006) Dairy products and ovarian cancer: a pooled analysis of 12 cohort studies. Cancer Epidemiol Biomarkers Prev 15, 364-372.

8. Guo, J., Astrup, A., Lovegrove, J.A., Gijsbers, L., Givens, D.I. and Soedamah-Muthu, S.S. (2017) Milk and dairy consumption and risk of cardiovascular diseases and all-cause mortality: dose-response meta-analysis of prospective cohort studies. Eur. J. Epidemiology 32, 269-287.

9. Horwath, C.C., Govan, C.H., Campbell, A.J., Busby, W. and Scott, V. (1995) Factors influencing milk and milk product consumption in young and elderly women with low calcium intakes. Nutrition Res. 15, 1735-1745.

10. Huncharek, M., Muscat, J. and Kupelnick, B. (2008) Dairy products, dietary calcium and vitamin D intake as risk factors for prostate cancer: a meta-analysis of 26,769 cases from 45 observational studies. Nutr Cancer 60, 421-441.

11. Imamura, F., Fretts, A., Marklund, M., Ardisson Korat, A.V., Yang, W.S., Lankinen, M., Qureshi, W., Helmer, C., Chen, C.A., Wong, K., Bassett, J.K., Murphy, R., Tintle, N., Yu, C.I., Brouwer, I.A., Chien, K.L., FrazierWood, A.C., del Gobbo, L.C., Djoussé, L., Geleijnse, J.M., Giles, G.G., de Goede, J., Gudnason, V., Harris, W.S., Hodge, A., Hu, F., Koulman, A., Laakso, M., Lind, L., Lin, H.J., McKnight, B., Rajaobelina, K., Risérus, U., Robinson, J.G., Samieri, C., Siscovick, D.S., Soedamah-Muthu, S.S., Sotoodehnia, N., Sun, Q., Tsai, M.Y., Uusitupa, M., Wagenknecht, L.E., Wareham, N.J., Wu, J.H., Micha, R., Forouhi, N.G., Lemaitre, R.N. and Mozaffarian, D. (2018) Fatty acid biomarkers of dairy fat consumption and incidence of type 2 diabetes: A pooled analysis of prospective cohort studies. PLOS Medicine, https://doi.org/10.1371/journal.pmed.1002670

12. Jeyaraman, M.M., Abou-Setta, A.M., Grant, L., Farshidfar, F., Copstein, L., Lys, J., Gottschalk, T., Desautels, D., Czaykowski, P., Pitz, M. and Zarychanski, R. (2019) Dairy product consumption and development of cancer: an overview of reviews. BMJ Open 9:e023625. doi:10.1136/

13. Lanou, A.J., Berkow, S.E. and Barnard, N.D. (2005) Calcium, dairy products, and bone health in children and young adults: a reevaluation of the evidence. Pediatrics 115, https://pediatrics.aappublications.org/content/ $115 / 3 / 736$ ?download $=$ true

14. Larsson, S. C., Orsini, N. and Wolk, A. (2006) Milk, milk products and lactose intake and ovarian cancer risk: a meta-analysis of epidemiological studies. Int J Cancer 118, 431-441.

15. Liu, J., Tang, W. and Sang, L. et al. (2015) Milk, yogurt, and lactose intake and ovarian cancer risk: a metaanalysis. Nutr. Cancer 67, 68-72.

16. Maruyama, K., Oshima, T. and Ohyama, K. (2010) Exposure to exogenous estrogen through intake of commercial milk produced from pregnant cows. Pediatr Int 52, 33-38.

17. Michaëlsson, K., Wolk, A., Langenskiöld, S., Lemming, E.W., Melhus, H. and Byberg, L. (2014) Milk intake and risk of mortality and fractures in women and men: cohort studies. BMJ 349, doi: https://doi.org/10.1136/ bmj.g6015

18. Moorman, P.G. \& Terry, P.D. (2004). Consumption of dairy products and the risk of breast cancer: a review of the literature. Am. J. Clin. Nutr., 80: 5-14.

19. Parodi, P.W. (2005). Dairy product consumption and the risk of breast cancer. J. Am. Coll. Nutr., 24: 556s$568 \mathrm{~s}$.

20. Qin, L. Q., Hy, X. and Wang, P. Y. et al. (2007) Milk consumption is a risk factor for prostate cancer in Western countries: evidence from cohort studies. Asia Pac J Clin Nutr 16, 467-476.

21. Qin, L. Q., Wang, P. Y. and Kaneko, T. et al. (2004) Estrogen: one of the risk factors in milk for prostate cancer. Med Hypotheses 62, 133-142.

22. Renehan, A. G., Zwahlen, M. and Minder, C. et al. (2004) Insulin-like growth factor (IGF)-I, IGF binding protein-3, and cancer risk: systematic review and meta-regression analysis. Lancet 363, 1346-1353.

23. Schwingshackl, L., Hoffmann, G., Schwedhelm, C., Kalle-Uhlmann, T., Missbach, B., Knuppel, S. and Boeing, H. (2016) Consumption of dairy products in relation to changes in anthropometric variables in adult populations: a systematic review and meta-analysis of cohort studies. PLoS One 11, e0157461, doi: 10.1371/ journal.pone. 0157461 
24. Thorning, T.K., Raben, A., Tholstrup, T., Soedamah-Muthu, S.S., Givens, I. and Astrup, A. (2016) Milk and dairy products: good or bad for human health? An assessment of the totality of scientific evidence. Food Nutrition Res. 60: 32527 -http://dx.doi.org/10.3402/fnr.v60.32527

25. Weaver, C., Wijesinha-Bettoni, R., McMohan, D. and Spence, L. (2013) Milk and dairy products as part of the diet. In Milk and Dairy Products in Human Nutrition (Muehlhoff, E., Bennett, A. and McMohan, D. eds.), Publisher- FAO of United Nations, Rome, 2013).

26. Yang, Y., Wang, X., Yao, Q., Qin, L. and Xu, C. (2016) Dairy product, calcium intake and lung cancer risk: a systematic review with meta-analysis. Science Reports 6, 20624; doi: 10.1038/srep20624 